\title{
Potential serotype coverage of three pneumococcal conjugate vaccines against invasive pneumococcal infection in Italian children
}

\author{
Chiara Azzari a,b,*, Maria Moriondo a,b, Martina Cortimiglia ${ }^{\mathrm{a}, \mathrm{b}}$, Claudia Valleriani ${ }^{\mathrm{a}, \mathrm{b}}$, \\ Clementina Canessa ${ }^{a, b}$, Giuseppe Indolfi ${ }^{a, b}$, Silvia Ricci ${ }^{a, b}, F^{2}$. \\ Massimo Resti ${ }^{\mathrm{a}, \mathrm{b}}$, the Italian group for the study of Invasive Pneumococcal Disease ${ }^{1}$ \\ a Department of Science for Women and Child Health, University of Florence, Viale Pieraccini 24, 50132 Firenze, Italy \\ ${ }^{\mathrm{b}}$ Anna Meyer Children Hospital, Florence, Viale Pieraccini 24, 50132 Firenze, Italy
}

\section{A R T I C L E I N F O}

\section{Article history:}

Received 30 August 2011

Received in revised form 9 November 2011

Accepted 3 December 2011

Available online 14 December 2011

\section{Keywords:}

Streptococcus pneumoniae

Potential coverage

PCV7

PCV10

PCV13

Serotype

Realtime PCR

\begin{abstract}
A B S T R A C T
Background and aim of the work: Since the introduction of the 7-valent vaccine, invasive pneumococcal disease have greatly decreased; however, changes in the distribution of pneumococcal serotypes have recently highlighted the need for vaccines with wider coverage. The aim of the work was to assess the potential serotype coverage of three pneumococcal conjugate vaccines (7-, 10- and 13-valent) against bacteremic pneumococcal pneumonia and meningitis/sepsis in Italian children.

Patients and methods: We determined pneumococcal serotypes in immunocompetent patients who had been admitted to hospital with suspicion of invasive bacterial disease and had confirmed bacteremic pneumococcal pneumonia or meningitis/sepsis determined by molecular detection of Streptococcus pneumoniae in a normally sterile site. Positive samples were serotyped using Realtime-PCR.

Results: Between April 2008 and March 2011, a total of 144 patients (age median 4.1 years; Interquartile range 1.8-5.6) with pneumococcal meningitis/sepsis $(n=43)$ or pneumonia $(n=101)$ from 83 participating centers located in 19 of 20 Italian regions were serotyped. The 10 most prevalent serotypes were 1 (29.9\%), 3 (16.0\%), 19A (13.2\%), 7F (8.3\%), 5 (4.2\%), 14 (4.2\%), 6A (3.5\%), 6B (3.5\%), 18C (3.5\%), 19F (3.5\%). Overall, serotype coverage for PCV-7, -10 and -13 were respectively $19.4 \%, 61.8 \%$ and $94.4 \%$ with no statistical difference between pneumonia and meningitis/sepsis. Potential coverage was similar for children $0-2$ or $2-5$ years of age. Cultures resulted positive in $35 / 99$ (35.4\%) samples simultaneously obtained for both culture and RT-PCR.

Conclusion: These findings indicate that increasing the potential serotype coverage by introducing PCV13 in the vaccination schedule for infancy could provide substantial added benefit for protection from pneumococcal pneumonia or meningitis/sepsis in Italy in children below 2 years as well in older children. The importance of molecular methods for diagnosis and serotyping of invasive pneumococcal disease was confirmed.
\end{abstract}

(C) 2012 Published by Elsevier Ltd.

\section{Introduction}

Streptococcus pneumoniae is the leading cause of community acquired invasive bacterial infections in young children and also

Abbreviations: IPD, Invasive pneumococcal disease; PCV, Pneumococcal conjugate vaccine; IQR, Interquartile range; RT-PCR, Realtime PCR.

* Corresponding author at: Immunology Department, Anna Meyer Children Hospital, Viale Pieraccini 24, 50132 Firenze, Italy. Tel.: +39 055 5662542; fax: +39055 4221012

E-mail addresses: c.azzari@meyer.it (C. Azzari),m.moriondo@meyer.it (M. Moriondo),m.cortimiglia@meyer.it (M. Cortimiglia), c.valleriani@meyer.it (C. Valleriani), clecle81@hotmail.com (C. Canessa), g.indolfi@meyer.it (G. Indolfi), silviaricci-85@hotmail.it (S. Ricci), maurizio.demartino@unifi.it (M. de Martino), m.resti@meyer.it (M. Resti).

1 See Appendix A. causes substantial morbidity and mortality in elderly patients [1]. More than 90 serotypes exist, but only a subset is associated with invasive disease [2].

Vaccines based on pneumococcal capsule polysaccharides alone are not effective for infants under 2 years of age [3] and their efficacy on older people is under debate [4]. Several pneumococcal conjugate vaccines (PCVs) were developed over the last decade to provide effective vaccine protection especially in young children. The conjugate vaccines have proven effective for reducing carriage and protecting against invasive disease [5,6]. However, there is evidence that serotypes not covered by the vaccine are increasing in incidence $[7,8]$, either for serotype shift facilitated by vaccination or for the well known secular trend of serotypes $[9,10]$.

In 2000 a PCV formulated with polysaccharide antigens from the seven serotypes isolated most frequently in north America (PCV7) was initially used for routine immunization of children in the USA. 


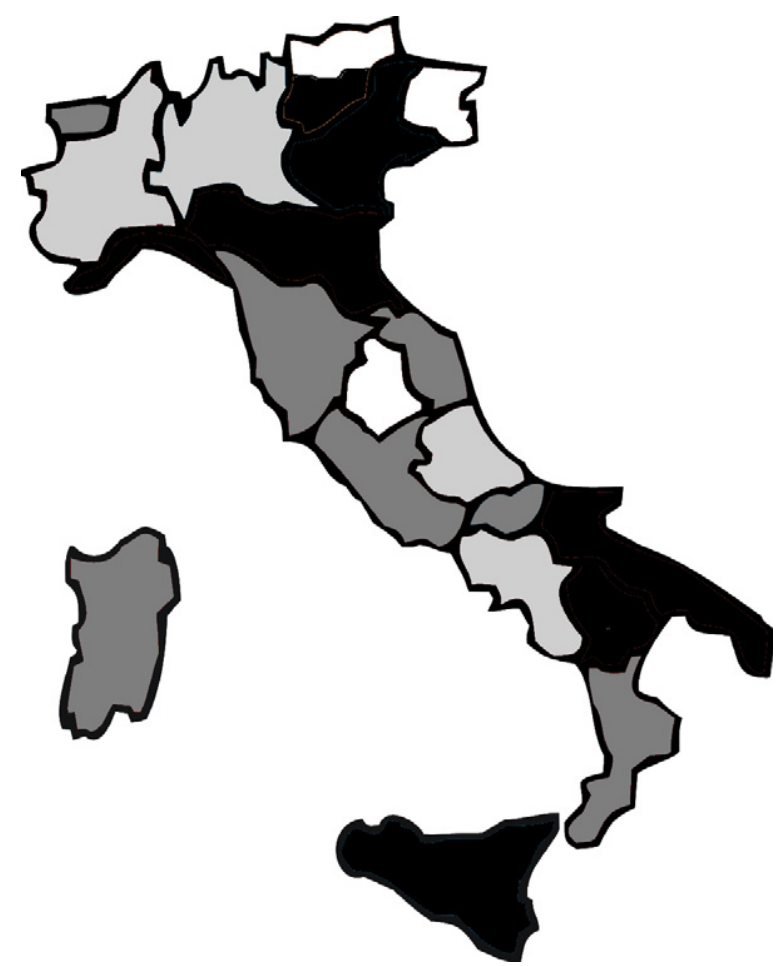

Fig. 1. Vaccination (PCV7) coverage in different Italian regions. Black: high coverage (>60\% vaccinated children); dark grey: medium coverage (30-60\% vaccinated children), light grey: low coverage ( $<30 \%$ vaccinated children); white: unknown coverage.

To date more than 100 million doses have been distributed worldwide. The European Medicines Agency (EMEA) granted a license for PCV7 in 2001. The Italian Ministry of Health initially recommended PCV7 for children at high risk, subsequently including it in the National Vaccination Plan for 2005-2007.

However PCV7 had been progressively introduced, since 2003, in some Italian regions, with different strategies according to epidemiological, organizational, and financial criteria.

In Liguria, for instance, PCV7 was recommended free-of-charge to all children in the first year of life already in 2003, and vaccine coverage rapidly increased to over $80 \%$ in 2004 [11]. On the contrary, other Administrative Regions, such as Lombardia did not recommend mass vaccination till July 2010 . This scenario has undoubtedly contributed to jeopardize vaccination coverage rates of the different Italian geographical areas [12] which would be expected to influence the number of the vaccine-preventable cases and cost effectiveness. PCV7 uptake in Italy was $50-60 \%$ over the period 2005-2007 based on international reports and reports from the National Department of Health (ICONA 2008) [13,14]; however, according to the ICONA 2008 survey, a great difference in PVC7 coverage was present among different Italian regions, ranging between 20 and 27\% respectively for Campania (South) and Lombardia (North) up to 94.3-95.2\% respectively for Basilicata (South) and Emilia-Romagna (North) [14] (Fig. 1).

Two new PCVs containing additional serotypes have been developed: the 10-valent PCV conjugated to Haemophilus influenzae glycoprotein-D, which adds serotypes 1,5 , and 7F (PCV10); and a CRM197-conjugated 13-valent PCV, which adds serotypes 1, 3, 5, 6A, 7F, and 19A (PCV13). In Italy, PCV13 will be deployed in many Regions in substitution of PCV7 [15,16].

The planning of a successful vaccination program requires information about potential coverage. The limited coverage provided by available PCVs with respect to the total number of serotypes emphasizes the importance of monitoring for emergence of non-vaccine serotypes. Differences in the pathologic potential of various serotypes should also be considered [2].

The aim of the present work was to assess the potential serotype coverage of three pneumococcal conjugate vaccines (PCV7, PCV10 and PCV13) against pneumococcal pneumonia and meningitis in Italian children.

\section{Patients and methods}

\subsection{Patients}

The study was designed to include, within an active surveillance program, all children $0-16$ years with a confirmed diagnosis of meningitis/sepsis (the most severe IPD) or bacteremic pneumonia (the most common IPD). Only patients admitted to Paediatric Hospitals or Paediatric wards of general hospitals in Italy from April 2008 through March 2011 were considered. Hospitals from all Italian regions were invited to participate.

IPD was defined as clinical suspicion of bacterial disease (pneumonia, meningitis/sepsis) [17-21] and laboratory-confirmation of the presence of $S$. pneumoniae in a normally sterile site (blood, cerebrospinal fluid or pleural fluid) as previously described [20].

Patients with severe concomitant disease (neoplasia, immunodepression) or nosocomial acquired infections were excluded. In order to select for community acquired disease, children who had been admitted to hospital, had been outpatients or had attended an emergency room in the previous 14 days were excluded from the study. Informed written consent was obtained from all parents or guardians before initiation of the study. The study was approved by the local ethics committee.

We recorded data on anti-pneumococcal vaccine status, including the number of doses received. Children were considered fully vaccinated if they had completed the national vaccination schedule, including 3 doses of the 7-valent conjugate anti-pneumococcal vaccine (PCV7) at 3,5 and 12 months of age, or two doses between the first and the second year of life, or a single dose after the second year of life. Children were considered incompletely vaccinated if they had started but not completed the vaccine schedule.

Accordingly to the coverage rate for PCV7 reported in ICONA 2008 surveillance report [14] Italian regions were divided in: low-coverage regions (0-33\% coverage), medium coverage regions (31-60\% coverage) and high coverage regions (61-100\% coverage). The distribution over the country is shown in Fig. 1.

\subsection{Sample handling}

Whole blood was obtained from all children as soon as possible after hospital admission. Samples for molecular tests were sent at room temperature to the central Laboratory (Immunology Laboratory, Anna Meyer Children's University Hospital, Florence, Italy) using an overnight freepost carrier and molecular tests were performed within $2 \mathrm{~h}$ of delivery; $200 \mu \mathrm{l}$ of whole blood were used for both diagnosis and serotyping by Realtime-PCR (RT-PCR). Clinicians were allowed to choose when to also request cultures from blood or cerebrospinal fluid (CSF). For culture purposes, blood or CSF samples were immediately sent to the local laboratory and procedures established by each hospital for these tests were used.

\subsection{Diagnosis and serotyping of IPD}

Diagnosis of laboratory-confirmed IPD was based on RT-PCR results for the lytA gene as previously described [20,22]. A sample was considered negative if there was no increase in fluorescent signal before RT-PCR cycle 45 . We had previously confirmed the specificity of PCR using whole blood samples drawn from carrier or non-carrier healthy controls [20,23]. 
Table 1

Primer and probe sets for pneumococcal serotyping by Realtime PCR.

\begin{tabular}{|c|c|c|c|}
\hline Serotype & Forward primer & Reverse primer & Probe \\
\hline 2 & TTATGGACTGGCTGATGGTTCTC & AAATCCTGACCCAATAATAGCCTTT & AGGTCAACGTATTGGAACTCTTAGAAATTGGGAAA \\
\hline $11 \mathrm{AD}$ & AGCTATCCTTTAGGCATTCCGTTA & TCCCGTTGGCTTAGATATGTGTT & TTGAAACACTAGATGAACTGGCAAACCT \\
\hline $16 \mathrm{~F}$ & CAGGCGAAAAGCGAGCAT & TGGGTTCCCCTCATCTACGTT & TGCTTTGGTAGCTTGTATGAGTGC \\
\hline $17 \mathrm{~F}$ & CTTAGCGTACGTTCTTCGTATGCTA & CCCGTACTCGGAAGCAAAAC & TCTAAGAGAGCTACTGAAACACTTTGTGC \\
\hline 21 & GGTTTAAATATCGCTCCGGGTAT & CAAAAAAAGGGCTTGTAGACGAA & TGTGAATTGGACACGTTATGGAGC \\
\hline $23 \mathrm{~A}$ & GGGAATTGGCACTCTTCTGAAT & GATCGGCAAATGTTGAAACCA & TTGGCGGTAAACAATTAAGGCGT \\
\hline 23B & TTGAAGAAATTGCTCCAGAAACAT & CCAAAAGACTAGCCTCAACCACTAA & TAGAGCTATTTATCTTTCGTGGTTTT \\
\hline 29 & TTCGAGTTGTGCCGTTTTTACA & GGCGTACCCACCTCTAAAATTTT & AGGAGTACGCAGAGAAAAGACTAGGATTCAA \\
\hline
\end{tabular}

All samples were serotyped using RT-PCR as previously described [20,22]. Twenty-nine primer/probe sets targeting different regions of the CpsA gene were used, each specific for 29 different serotypes. Twenty-one primer-probe sets were previously published $[20,22]$; the sequences of the 8 additional primer probe sets are shown in Table 1 . If no increase in fluorescent signal was observed after 45 cycles for any of the serotype-specific primer/probe sets in spite of a positive result with both RT-PCR (lytA gene) and end-point PCR (CpsA gene) [22], the sample was considered non-typeable with the serotype-specific primers in Real-time PCR. Pneumococcal serotypes were classified as covered by PCV-7, PCV-10 or PCV-13 vaccines if they were included in the 7-valent (4, $6 \mathrm{~B}, 9 \mathrm{~V}, 14,18 \mathrm{C}, 19 \mathrm{~F}, 23 \mathrm{~F}), 10$-valent (7-valent with the addition of 1,5 and $7 F$ ) or 13 -valent (10-valent with further addition of $3,6 \mathrm{~A}$ and 19A) conjugate vaccines, respectively. The potential coverage of the 3 available conjugate vaccines has been evaluated according to the disease; moreover, since a campaign for vaccination of children 2-5 years old has been proposed in some Italian regions, the potential coverage has been evaluated according to different age.

\subsection{Statistical analysis}

Two tailed $p$ values were used and $p$ values $<0.05$ were considered statistically significant. Results were expressed as means and standard deviations (SD) or as median and interquartile range (IQR) as appropriate. Data were processed with the SPSSX statistical package (SPSS 11.0, SPSS Inc, Chicago, IL).

\section{Results}

\subsection{Diagnosis of IPD}

We identified a total of 153 patients with bacteremic pneumococcal pneumonia $(n=104)$ or meningitis/sepsis $(n=49)$ from 83 participating centers located among 19 of the 20 Italian regions, representing $99.8 \%$ of the Italian population below the age of 16 years.

Among the 20 Italian regions, 4 regions had low-coverage for PCV7, 7 regions had medium coverage for PCV7, 6 regions and one autonomous province had high coverage for PCV7. Data on PCV7 coverage were not available for 2 regions and one autonomous province. Geographical distribution was not uniform (Fig. 1) so that PCV7 high-coverage regions are present both in the north and in the south of Italy. Twenty-five samples were obtained from low-coverage regions, 80 samples from medium-coverage regions and 48 from high coverage regions. The only region which did not include any patient has 18,807 residents below 16 years of age that is $0.2 \%$ of Italian population $(9,044,793) 0-16$ years (Italian National Institute of Statistic, ISTAT, 2010 http://demo.istat.it/; accessed 6.11.11).

Median (IQR) age was $3.3(1.7-5.6)$ years. The gender ratio $\mathrm{M} / \mathrm{F}$ was 1.12 . Vaccination records indicated that $40.5 \%(62 / 153)$ had completed the vaccination schedule and were fully vaccinated; among them 1 child had completed the vaccination schedule with

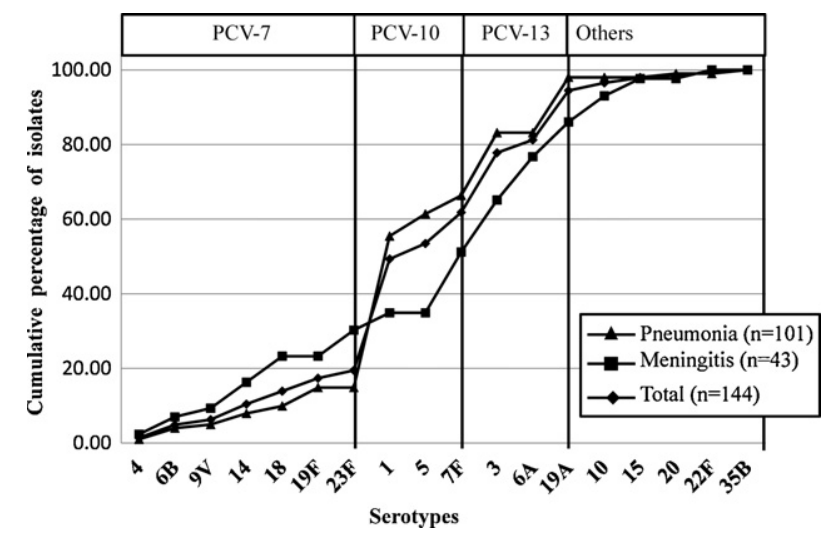

Fig. 2. Incidence of various serotypes as a cumulative percentage according to type of invasive pneumococcal disease in Italian children.

one dose of PCV13; 7.2\%(11/153) had received at least one dose but not the full course ( 2 of them had received 1 dose of PCV13) and $43.8 \%(67 / 153)$ had not been vaccinated. Data regarding vaccination was not available for $8.5 \%$ (13/153) patients.

In 99 samples simultaneously obtained for both culture and RTPCR and positive for $S$. pneumoniae by RT-PCR, cultures resulted positive in 35/99 (35.4\%) children. Molecular methods appeared 2.8 times more sensitive than cultural methods $\left(p<10^{-6}\right.$; OR and $95 \%$ confidence limits not calculable). Pathogens different from $S$. pneumoniae were never found, in any of the samples positive for $S$. pneumoniae by RT-PCR with the exception of one sample, positive for Staphilococcus epidermidis by culture and reported as contaminated by the laboratory microbiologist.

\subsection{Serotype distribution}

Individuation of pneumococcal serotype was obtained in 144/153 (94.1\%) patients (101 pneumonia, 43 meningitis/sepsis; median age 3.3, IQR 1.8-5.6). IPD remained non-typeable in 9/153 (5.9\%) patients because of sample paucity (1 sample) or because negativity to all available primer/probe sets (8/153, 5.2\% samples). The 10 most prevalent serotypes were 1 (43/144, 29.9\%), 3 (23/144, 16.0\%), 19A (19/144, 13.2\%), 7F (12/144, 8.3\%), 5 (6/144, 4.2\%), 14 (6/144, 4.2\%), 6A (5/144, 3.5\%), 6B (5/144, 3.5\%), 18C (5/144, 3.5\%), $19 \mathrm{~F}(5 / 144,3.5 \%)$. The distribution of the serotypes identified in these patients according to underlying disease is shown in Fig. 2. Overall, serotype coverage for PCV-7, -10 and -13 were $19.4 \%, 61.8 \%$ and $94.4 \%$. In order to determine the impact of age on potential coverage we have presented serotype distribution according to patient age (Fig. 3): serotype distribution was similar in the two groups of patients.

The percentage of vaccinated (fully or partially vaccinated) children decreased, as expected, with age, going from $59.1 \%$ in children below 2 years of age, to $56.9 \%$ in children $2-5$ years of age, to $12.5 \%$ in children over 9 years of age. 


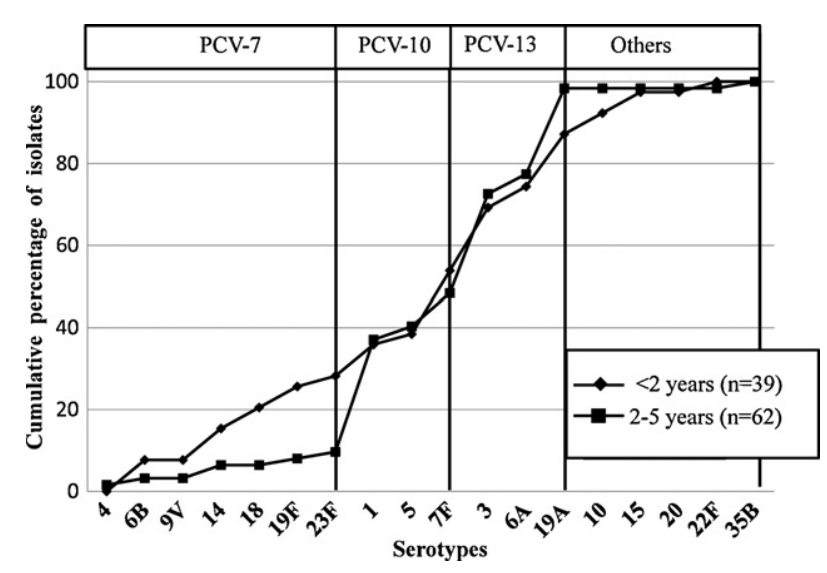

Fig. 3. Incidence of various serotypes as a cumulative percentage according to age in Italian children with invasive pneumococcal disease.

PCV7 serotypes were found in 7/25 (28.0\%) serotyped samples from low-coverage regions, in 15/71 (21.1\%) serotyped samples from medium-coverage regions and in 5/45 (11.1\%) serotyped samples from high coverage regions.

No case of IPD due to PCV7 serotypes was recorded in children who had been fully or partially vaccinated.

\section{Discussion}

Knowing the present distribution of pneumococcal serotypes is essential to plan and monitor mass vaccination. Our study, performed on a large population of children distributed throughout Italy shows that, at present, less than 20\% IPD are due to serotypes included in PCV7, and the potential coverage obtainable by 10 valent or 13 -valent formulation is respectively $61.8 \%$ and $94.4 \%$.

The comparison between Realtime PCR and culture sensitivity was beyond the aim of the study; however the data obtained in this study confirm $[19,20]$ the higher sensitivity of molecular methods compared to culture methods and underline the essential role of molecular methods in surveillance studies.

Our survey reveals a change in the distribution of pneumococcal serotypes in Italy since the 7-valent vaccine was introduced. Surveys conducted before PCV7 was introduced in Italy had revealed higher potential coverage for that vaccine, both in children $<2$ years and $<5$ years [12,24-28]. Actually, prior to the introduction of PCV7, active surveillance carried out in 10 hospitals demonstrated that PCV-7, PCV-10 and PCV-13 could have prevented, respectively, 70, 80 and $87 \%$ cases in children $<2$ years old [24]. Regarding children $<5$ years old, published results demonstrated that PCV-7, PCV-10 and PCV-13 could have prevented respectively $67-80 \%, 78-88 \%$ and $87-100 \%$ cases in the period between 1997 and 2003 [12,24-27]. Unfortunately, since in Italy surveillance of invasive bacterial disease was, up to 2008 , only focused on meningitis, larger studies included only that disease, and few information on other IPD different from meningitis were available.

The present work shows that, in the last 3 years, most frequent serotypes are 1, 3 and 19A in IPD patients with pneumonia and $7 \mathrm{~F}, 3$ and $6 \mathrm{~A}$ in patients with meningitis (none of those serotypes is included in the PCV7 formulation). Over $50 \%$ of meningitis and over $80 \%$ of all pneumonia cases are due to serotypes which are not included in the PCV7 but are included in the 13-valent formulation. As expected, there is a trend to a lower rate of PCV7 serotypes in regions with high PCV coverage rate; even if data does not reach significant differences, the rate of infections due to PCV7 serotypes is almost double in regions with medium PCV7-coverage and almost three times in low coverage regions when compared to high PCV7-coverage regions.
Similar findings have been reported across Europe. The most common serotypes associated with IPD before the introduction of PCV7 were 14, 6B, 19F, and 23F, which are all covered by PCV7. At that time, the mean increases in potential coverage for PCV10 and PCV13 were $7 \%$ and $16 \%$, respectively, for children $<2$ years of age [29]. Currently, data from countries constituting approximately $60 \%$ of the European population reveal that the most common IPD serotypes after the introduction of PCV7 are 1, 19A, 3, 6A, and 7F [29]. In particular in Portugal [30] and France [31] the 3 leading serotypes are $1,19 \mathrm{~A}$ and $7 \mathrm{~F}$, with similar percentages in the two countries (24-26\%, 20-25\% and $12-14 \%$ respectively). Different from those data, our results show a higher prevalence of serotype 3; this is maybe due to the fact that Portuguese and French data were obtained between 2006 and 2009, while our data are more recent. Actually, in our Italian patients, the prevalence of serotype 3 was about $8 \%$ in 2008 but it increased up to $22.0 \%$ in the last year.

A mild increase of non-vaccine serotypes has been described after mass vaccination [32] and is associated with a decrease in the number of cases due to vaccine serotypes [5,11].

However this does not seem to be the case in Italy where vaccination has been limited to some regions and only from July 2010 is offered free of charge to all children below 1 year of age. Actually the rate of vaccinated children seems to be less than $50 \%$ in the last 3 years and undoubtedly lower in the previous years. Therefore, the high frequency of serotypes 1,3 and 19A shown in the present work and the increase of those serotypes compared to 5-10 years ago [12,24-27], cannot be completely ascribed to the immunological pressure caused by the PCV7 vaccination. A spontaneous increase of some serotypes, as demonstrated in the recent pre-vaccination era [33] and the secular trend demonstrated for different serotypes in the last 50 years $[9,10]$, may probably contribute to the trend observed in Italy.

Our data indicates that a shift to vaccines that include more serotypes is necessary in Italy. PCV10 would provide potential coverage for about $62 \%$ of the serotypes identified, while PCV13 would provide coverage of more than $94 \%$ ( $86 \%$ for meningitis/sepsis and nearly $100 \%$ for pneumonia).

There is no doubt that mass vaccination of children below one year of age is worthy; decision regarding vaccination of children between 1 and 5 years will be taken at the regional level. Similarly, catch-up of children previously vaccinated with PCV7 in order to expand their protection needs to be evaluated. First of all, the evaluation of serotype distribution in this age class is mandatory, in order to consider which coverage could be obtained with different vaccine formulations. Our data demonstrate that pneumococcal serotype distribution in children between 2 and 5 years does not differ from epidemiology demonstrated in younger children and that PCV13 could prevent over 90\% IPD in that cohort as it does in children below 2 years. Other studies [34] evaluating the cost benefit analysis for that age are now in progress in our laboratory.

\section{Conclusions}

Our results strongly suggest that introduction of PCV13 could have a significant added benefit in reducing the burden of pneumococcal disease in Italy. Molecular methods are essential tools in the monitoring of pneumococcal serotype distribution. Continuous surveillance of pneumococcal serotype distribution is mandatory to plan and monitor the effect of pneumococcal vaccination and promptly catch serotype shift.

\section{Acknowledgments}

We are sincerely grateful to Francesca Romano and Giusy Mangone for their technical support. The research has been partially 
supported by the Italian Department of Health; Italian Center for Disease Control and Prevention (grant 117-19.01.07-\#6728 to C.A.). The authors do not have any conflict of interest.

\section{Appendix A.}

Italian group for the study of Invasive Pneumococcal Disease: Agostiniani R., Pistoia; Allievi P., Garbagnate Milanese; Allù G., Ragusa; Amigoni A., Ravenna; Bernardi P., Bologna; Bernardini R., Empoli; Biban P., Borgotrento; Bigi M., Rimini; Boldrini A., Pisa; Bossi G., Pavia; Bottone U., Massa; Cardinale A., Montevarchi; Cardona A., Foligno; Castronari R., Perugia; Celandroni A., Pontedera; Chiossi M., Colleselli P., Vicenza; Cuneo; Correra A., Napoli; D’Ascola G, Arezzo; D’Aquino A.; Udine; De Benedictis F.M., Ancona; Dini E., Firenze; Dollfus L., Terni; Domenici R., Lucca; Flacco V., Furbetta M., Perugia; Gaetti M.T., Jesi; Gagliardi L., Viareggio; Giani I., Montepulciano; Giglio P., Gubbio; Guala A., Verbania; Lanciano; Lanari M., Imola; Lippi F., Firenze; Lizzoli C., Magenta; Lombardi E., Firenze; Macchia PA, Pisa; Magnini M., Esine; Memmini G., Carrara; Mesirca P., Montebelluna; Micheletti E., Livorno; Migliozzi L., Senigallia; Nunziata F., Solofra; Pecile P., Udine; Pepe G., Gallipoli; Perferi G., Firenze; Peris A., Firenze; Perri P.F., Macerata; Pescollderungg L., Bolzano; Pezzati M., Firenze; Poggi G.M., Firenze; Prato R., Foggia; Principi N., Milano; Rapisardi G., Firenze; Regoli M., Firenze; Riva A., Brescia; Rizzo L., Portoferraio; Roman B., Vimercate; Toffolo A., Oderzo; Strano M., Borgo San Lorenzo; Trapani S., Firenze; Vasarri P., Prato; Vascotto M., Siena; Vecchi V., Rimini; Ventura A., Trieste; Verini M., Chieti; Zorzi C., Camposanpiero.

\section{References}

[1] Bridy-Pappas AE, Margolis MB, Center KJ, Isaacman DJ. Streptococcus pneumoniae: description of the pathogen, disease epidemiology, treatment, and prevention. Pharmacotherapy 2005;25:1193-212.

[2] Hausdorff WP, Feikin DR, Klugman KP. Epidemiological differences among pneumococcal serotypes. Lancet Infect Dis 2005;5:83-93.

[3] Ledwith M. Pneumococcal conjugate vaccine. Curr Opin Pediatr 2001;13:70-4.

[4] 23-valent pneumococcal polysaccharide vaccine. WHO position paper. Wkly Epidemiol Rec 2008;83:373-84, http://www.who.int/wer.

[5] Whitney CG, Farley MM, Hadler J, Harrison LH, Bennett NM, Lynfield R, et al. Decline in invasive pneumococcal disease after the introduction of protein-polysaccharide conjugate vaccine for the active bacterial core surveillance of the emerging infections program network. N Engl J Med 2003;348:1737-46.

[6] Grijalva CG, Nuorti JP, Arbogast PG, Martin SW, Edwards KM, Griffin MR. Decline in pneumonia admissions after routine childhood immunisation with pneumococcal conjugate vaccine in the USA: a time-series analysis. Lancet 2007;369:1179-86.

[7] Kyaw MH, Lynfield R, Schaffner W, Craig AS, Hadler J, Reingold A, et al. Active Bacterial Core Surveillance of the Emerging Infections Program Network. Effect of introduction of the pneumococcal conjugate vaccine on drug-resistant Streptococcus pneumoniae. N Engl J Med 2006;354:1455-63.

[8] Steenhoff AP, Shah SS, Ratner AJ, Patil SM, McGowan KL. Emergence of vaccinerelated pneumococcal serotypes as a cause of bacteremia. Clin Infect Dis 2006;42:907-14.

[9] Butler JC, Breiman RF, Lipman HB, Hofmann J, Facklam RR. Serotype distribution of Streptococcus pneumoniae infections among preschool children in the United States, 1978-1994: implications for development of a conjugate vaccine. J Infect Dis 1995;171:885-9.

[10] Finland M, Barnes MW. Changes in occurrence of capsular serotypes of Streptococcus pneumoniae at Boston City Hospital during selected years between 1935 and 1974. J Clin Microbiol 1977;5:154-66.

[11] Durando P, Crovari P, Ansaldi F, Sticchi L, Sticchi C, Turello V, et al. Universal childhood immunisation against Streptococcus pneumoniae: the five-year experience of Liguria Region, Italy. Vaccine 2009;27:3459-62.

[12] D'Ancona F, Salmaso S, Barale A, Boccia D, Lopalco PL, Rizzo C, et al. Incidence of vaccine preventable pneumococcal invasive infections and blood culture practices in Italy. Vaccine 2005;23:2494-500.
[13] De Carvalho Gomes H, Muscat M, Monnet DL, Giesecke J, Lopalco PL. Use of seven-valent pneumococcal conjugate vaccine (PCV7) in Europe, 2001-2007. Euro Surveill 2009;1:4, pii:19159.

[14] Istituto Superiore di Sanità. ICONA 2008: national vaccination coverage survey among children and adolescents. ICONA Working Group; 2009, http://www.iss.it/binary/publ/cont/09_29_web.pdf [accessed 6.11.11].

[15] Alicino $C$, Iudici R, Alberti $M$, Durando P. The dangerous synergism between influenza and Streptococcus pneumoniae and innovative perspectives of vaccine prevention. J Prev Med Hyg 2011;52(September (3)): 102-6.

[16] Boccalini S, Azzari C, Resti M, Valleriani C, Cortimiglia M, Tiscione E, et al. Economic and clinical evaluation of a catch-up dose of 13 -valent pneumococcal conjugate vaccine in children already immunized with three doses of the 7-valent vaccine in Italy. Vaccine 2011. October 18 [Epub ahead of print].

[17] Overturf GD. Defining bacterial meningitis and other infections of the central nervous system. Pediatr Crit Care Med 2005;6:S14-8.

[18] Russell JA. Management of sepsis. N Engl J Med 2006;355:1699-713.

[19] Azzari C, Moriondo M, Indolfi G, Massai C, Becciolini L, de Martino M, et al. Molecular detection methods and serotyping performed directly on clinical samples improve diagnostic sensitivity and reveal increased incidence of invasive disease by Streptococcus pneumoniae in Italian children. J Med Microbiol 2008;57:1205-12.

[20] Resti M, Moriondo M, Cortimiglia M, Indolfi G, Canessa C, Becciolini L, et al. Community-acquired bacteremic pneumococcal pneumonia in children: diagnosis and serotyping by real-time polymerase chain reaction using blood samples. Clin Infect Dis 2010;51:1042-9.

[21] Randle E, Ninis N, Inwald D. Invasive pneumococcal disease. Arch Dis Child Educ Pract Ed 2011;96(October (5)):183-90, doi:10.1136/adc.2010.191718.

[22] Azzari C, Moriondo M, Indolfi G, Cortimiglia M, Canessa C, Becciolini L, et al. Realtime PCR is more sensitive than multiplex PCR for diagnosis and serotyping in children with culture negative pneumococcal invasive disease. PLoS One 2010;5(2):e9282.

[23] Azzari C, Cortimiglia M, Moriondo M, Canessa C, Lippi F, Ghiori F, et al. Pneumococcal DNA is not detectable in the blood of healthy carrier children by real-time PCR targeting the lytA gene. J Med Microbiol 2011;60: 710-4.

[24] Tarallo L, Tancredi F, Schito G, Marchese A, Bella A, Italian Pneumonet Group. Active surveillance of Streptococcus pneumoniae bacteremia in Italian children. Vaccine 2006;24:6938-43.

[25] Pantosti A, D’Ambrosio F, Tarasi A, Recchia S, Orefici G, Mastrantonio P. Antibiotic susceptibility and serotype distribution of Streptococcus pneumoniae causing meningitis in Italy, 1997-1999. Clin Infect Dis 2000;31: 1373-9.

[26] Pantosti A, Boccia D, D’Ambrosio F, Recchia S, Orefici G, Moro ML. Inferring the potential success of pneumococcal vaccination in Italy: serotypes and antibiotic resistance of Streptococcus pneumoniae isolates from invasive diseases. Microb Drug Resist 2003;9(Suppl. 1):S61-8.

[27] Gherardi G, D’Ambrosio F, Monaco M, Camilli R, De Florio L, D’Ancona F, et al. Population structure of invasive Streptococcus pneumoniae isolates in Italy prior to the implementation of the 7-valent conjugate vaccine (1999-2003). Eur J Clin Microbiol Infect Dis 2009;28:99-103.

[28] Principi N, Marchisio P. Epidemiology of Streptococcus pneumoniae in Italian children. Acta Paediatr Suppl 2000;89(December (435)):40-3.

[29] Isaacman DJ, McIntosh ED, Reinert RR. Burden of invasive pneumococcal disease and serotype distribution among Streptococcus pneumoniae isolates in young children in Europe: impact of the 7-valent pneumococcal conjugate vaccine and considerations for future conjugate vaccines. Int J Infect Dis 2010;14:e197-209.

[30] Aguiar SI, Brito MJ, Gonçalo-Marques J, Melo-Cristino J, Ramirez M. Serotypes $1,7 \mathrm{~F}$ and $19 \mathrm{~A}$ became the leading causes of pediatric invasive pneumococcal infections in Portugal after 7 years of heptavalent conjugate vaccine use. Portugal Vaccine 2010;28:5167-73.

[31] Grall N, Hurmic O, Al Nakib M, Longo M, Poyart C, Ploy MC, et al. Epidemiology of Streptococcus pneumoniae in France before introduction of the PCV-13 vaccine. Eur J Clin Microbiol Infect Dis 2011. April 18 [Epub ahead of print].

[32] Byington CL, Samore MH, Stoddard GJ, Barlow S, Daly J, Korgenski K, et al. Temporal trends of invasive disease due to Streptococcus pneumoniae among children in the Intermountain West: emergence of nonvaccine serogroups. Clin Infect Dis 2005;41:21-9.

[33] Flamaing J, Verhaegen J, Vandeven J, Verbiest N, Peetermans WE. Pneumococcal bacteraemia in Belgium (1994 2004): the pre-conjugate vaccine era. J Antimicrob Chemother 2008;61:143-9.

[34] Boccalini S, Azzari C, Resti M, Valleriani C, Cortimiglia M, Tiscione E, et al. Economic and clinical evaluation of a catch-up dose of 13valent pneumococcal conjugate vaccine in children already immunized with three doses of the 7-valent vaccine in Italy. Vaccine 2011;29: 9521-8. 